08,09

\title{
Новые линии люминесценции в полученных методом химического газофазного осаждения наноалмазах
}

\author{
() В.Г. Голубев ${ }^{1}$, С.А. Грудинкин ${ }^{1,2, \uparrow}$, В.Ю. Давыдов ${ }^{1}$, А.Н. Смирнов ${ }^{1}$, Н.А. Феоктистов ${ }^{1}$ \\ ${ }^{1}$ Физико-технический институт им. А.Ф. Иофффе РАН, \\ Санкт-Петербург, Россия \\ ${ }^{2}$ Санкт-Петербургский национальный исследовательский университет \\ инфрормационных технологий, механики и оптики, \\ Санкт-Петербург, Россия \\ ฯ E-mail: grudink.gvg@mail.ioffe.ru \\ (Поступила в Редакцию 5 июня 2017 г.)
}

Исследованы спектральные характеристики обнаруженных линий фотолюминесценции наноалмазов, полученных методом реактивного ионного травления в кислородной плазме алмазных частиц, осажденных методом химического газофазного осаждения на кремниевую подложку. При комнатной температуре в видимой и инфракрасной областях спектра наблюдались узкие линии с полной шириной на полувысоте максимума в диапазоне 1-2nm при практически полном отсутствии широкополосного фонового сигнала фотолюминесценции. При понижении температуры линии сужались до $0.2-0.6 \mathrm{~nm}$ при $T=79 \mathrm{~K}$ и минимальная ширина линий составила $0.055 \mathrm{~nm}$ при $T=10 \mathrm{~K}$. С ростом температуры узкие линии сдвигались в длинноволновую область спектра и их интенсивность уменьшалась.

DOI: 10.21883/FTT.2017.12.45235.182

\section{1. Введение}

В последние годы большое внимание уделяется созданию и исследованию центров окраски в алмазе, излучающих в видимой и ближней ИК области спектра [1-7]. Благодаря таким свойствам алмаза как широкая запрещенная зона, высочайшая теплопроводность и твердость, химическая инертность, высокая температура Дебая, радиационная стойкость этот материал является исключительно удачной матрицей для центров окраски [8]. Такие центры обладают яркой и стабильной фотолюминесценцией (ФЛ) при комнатной температуре, узкой бесфононной линией (БФЛ) и короткими излучательными временами жизни ФЛ $[1,2,6]$. Это открывает возможность их применения в качестве однофотонных источников излучения в устройствах обработки квантовой информации и интегральной нанофотоники. Биосовместимость, химическая инертность, низкая токсичность и возможность химической функционализации поверхности наноалмазов открывает перспективу применения наноалмазов с центрами окраски для биомедицины [7]. В алмазных пленках и частицах, полученных методом химического газофазного осаждения (chemical vapor deposition-CVD), наибольший интерес прикован к следующим центрам окраски: кремний-вакансия ( $\mathrm{SiV})$ [9-20], Ni-содержащим [21-25], Cr-содержащим [26-28], азот-вакансия (NV) [29,30]. Недавно в спектрах ФЛ в алмазе, полученном методом CVD, обнаружены узкие линии ФЛ [31-33], структура и механизм формирования которых остаются не до конца установленными.

Для практического использования эмиттеров на основе центров окраски необходимо подавление фоно- вого сигнала ФЛ и уменьшение влияния безызлучательных каналов люминесценции, что может быть достигнуто в алмазе с низким содержанием структурных дефектов.

Данная работа посвящена обнаружению и исследованию спектральных характеристик узких линий ФЛ наноалмазов в видимом и ближнем ИК спектральном диапазоне в интервале температур 10-300 К. Наноалмазы получены методом реактивного ионного травления (РИТ) в кислородной плазме алмазных частиц, выращенных методом микроволнового химического газофазного осаждения (MWCVD) на кремнии [34]. Травление удаляет из алмазных частиц наиболее дефектные области, имеющие внутренние напряжения, которые ответственны за уширение рамановской линии и неоднородное уширение линий ФЛ центров окраски [34].

\section{2. Методика эксперимента}

Наноалмазы детонационного синтеза с характерным размером $\sim 4 \mathrm{~nm}$ использовались в качестве центров зародышеобразования и контролируемо наносились на кремниевую подложку методом аэрозольного распыления [35]. Деагломерированные детонационные наноалмазы получены в ФТИ им. А.Ф. Иоффе методом, описанном в [36]. Поверхностная плотность наноалмазов детонационного синтеза составляла $\sim 10^{6} \mathrm{~cm}^{-2}$. Параметры процесса MWCVD были следующие: микроволновая мощность - $600 \mathrm{~W}(2.45 \mathrm{GHz})$, температура подложки $-700^{\circ} \mathrm{C}$, расход водорода - $500 \mathrm{sccm}$, концентрация метана - 2.8\%, рабочее давление в реакторе - 15 Torr. Время роста частиц составляло около $40 \mathrm{~min}$. 
Травление алмазных частиц осуществлялось в кислородно-азотной смеси $\left(\mathrm{O}_{2}-20 \mathrm{vol} \%, \mathrm{~N}_{2}-80 \mathrm{vol} \%\right)$ при следующих параметрах: микроволновая мощность - $250 \mathrm{~W}(2.45 \mathrm{GHz})$, температура подложки - 500-600 ${ }^{\circ} \mathrm{C}$, расход кислородно-азотной смеси $100 \mathrm{sccm}$, рабочее давление в реакторе - 10 Torr, время травления - $30 \mathrm{~min}$. Подробное описание процессов роста, контроля размеров частиц в процессе роста и реактивного ионного травления изложены в работе [34]. Спектры ФЛ регистрировались от ансамблей наноалмазов, сформированных после травления исходных частиц.

Измерения методами комбинационного рассеяния света (КРС) и микрофотолюминесценции ( $\mu$-ФЛ) проводились на спектрометре Horiba Jobin Yvon T64000, оснащенном конфокальным микроскопом, охлаждаемой жидким азотом кремниевой ПЗС матрицей и дифракционной решеткой 1800 lines $/ \mathrm{mm}$. При измерениях использовалась длина волны возбуждающего света $532 \mathrm{~nm}$ $(2.33 \mathrm{eV})$. Спектры КРС измерялись при комнатной температуре в пятне $\sim 1 \mu \mathrm{m}$ на образце, полученном с использованием объектива $100 \times(\mathrm{NA}=0.9)$. Все КРС измерения проводились в режиме тройного монохроматора со сложением дисперсии, что обеспечивало максимальное спектральное разрешение $0.35 \mathrm{~cm}^{-1}$. Измерения низкотемпературной $\mu$-ФЛ проводились в гелиевой системе замкнутого цикла для микроскопии (Cryo Industries, Inc.), либо в приставке для микроскопии Linkam THMS600. Для фокусировки падающего пучка в пятно диаметром $\sim 2 \mu \mathrm{m}$ при ФЛ измерениях использовался объектив Mitutoyo $100 \times(\mathrm{NA}=0.50)$ с большим рабочим расстоянием.

\section{3. Экспериментальные результаты и их обсуждение}

Спектры ФЛ трех различных ансамблей наноалмазов (NDE1-NDE3), измеренных при комнатной температуре показаны на рис. 1. Интенсивности линий в спектрах нормированы на интенсивность линии центра $\mathrm{SiV}$ с максимумом на длине волны $738 \mathrm{~nm}$. Эта линия характерна для алмазов, полученных методом химического газофазного осаждения на кремний содержащие подложки. Центры окраски $\mathrm{SiV}$ введены в алмазные частицы в процессе их синтеза. Травление атомарным водородом кремниевой подложки обеспечивало поступление кремния в газовую смесь. Кремний из газовой фазы встраивался в решетку растущих алмазных частиц. Внедряясь в решетку алмаза, атомы $\mathrm{Si}$ стимулируют образование вакансий в соседних узлах решетки, что приводит к формированию точечных дефектов - оптически активных центров $\mathrm{SiV}$. Отметим, что существует также возможность контролируемого введения центров окраски $\mathrm{SiV}$ в алмаз путем добавления силана в рабочую газовую смесь как источника примесных атомов $\mathrm{Si}$ в процессе химического газофазного осаждения частиц

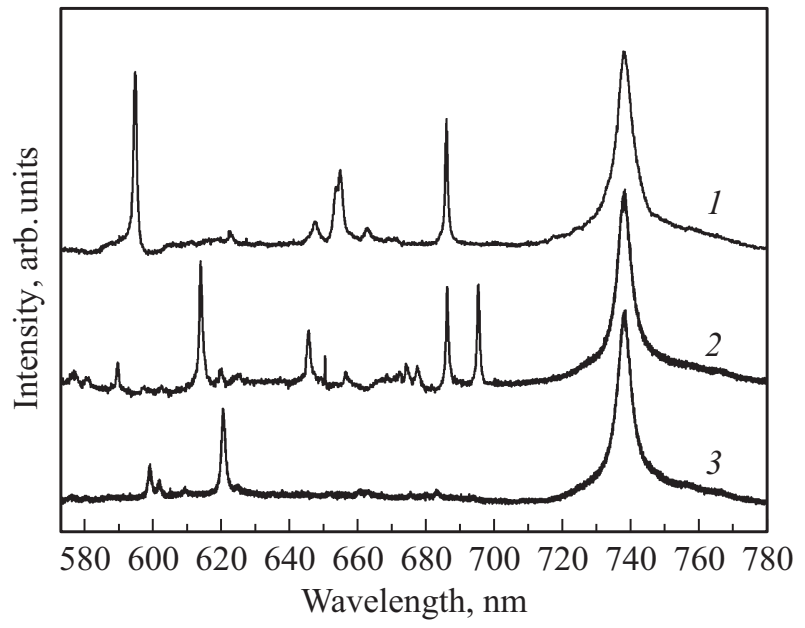

Рис. 1. Спектры ФЛ трех различных ансамблей наноалмазов ( 1 - NDE1, 2 - NDE2, 3 - NDE3), измеренные при $T=300 \mathrm{~K}$. Мощность возбуждающего лазера $0.4 \mathrm{~mW}$.

на подложки, не содержащей кремний (например, сапфир [37] и $\mathrm{AlN}$ [38]).

В спектрах ФЛ на рис. 1 наблюдается набор узких линий для каждого ансамбля наноалмазов. Спектральные положения максимумов наиболее интенсивных линий ФЛ зарегистрированы на длинах волн $594.5(1.16) \mathrm{nm}, 647.4(2.2) \mathrm{nm}$, дуплет $653.4 \mathrm{~nm} \mathrm{и}$ $654.8 \mathrm{~nm}, 685.87(1.3) \mathrm{nm}$ для ансамбля NDE1; $589.4 \mathrm{~nm}$, $613.8 \quad(1) \mathrm{nm}, \quad 645.3 \quad(1.3) \mathrm{nm}, \quad 686.17 \quad(0.85) \mathrm{nm}$ и 695.3 (1.05) для NDE2; 599 (1) nm, 601.6 (1.15) nm и 620.4 (4.2) nm для NDE3. В скобках указана FWHM для линий, в которых она надежно определялась. При комнатной температуре FWHM обнаруженных линий $(1-2 \mathrm{~nm})$ меньше, чем у БФЛ ансамбля центров окраски $\mathrm{SiV}[9,11,12,15,18,19,34], \mathrm{Cr}$-содержащих центров [26,27], и сравнима с шириной БФЛ одиночных центров окраски $\mathrm{SiV}$ в алмазе $[13,20]$. В спектрах исследованных наноалмазов практически отсутствует фоновой сигнал в виде широкой полосы ФЛ с максимумом в видимой области спектра, которая характерна для различных гетероэпитаксиальных алмазов $[10,11,15]$. Широкая полоса ФЛ связана с оптическими переходами внутри запрещенной зоны алмаза, обусловленными присутствием неупорядоченного $s p^{2}$-гибридизованного углерода [39]. Значительное уменьшение содержания $s p^{2}$-углерода и неупорядоченного $s p^{3}$-алмаза в исследуемых наноалмазах достигнуто селективным РИТ в кислородной плазме [34]. Низкая интенсивность фоновой ФЛ исследуемых наноалмазов является преимуществом в случае их применения в качестве твердотельной матрицы для однофотонных источников излучения.

На рис. 2 показаны фрагменты спектров ФЛ ансамбля наноалмазов (NDE1) при мощности излучения возбуждающего лазера $0.04 \mathrm{~mW}$ и $1 \mathrm{~mW}$. Спектры измерены при комнатной температуре. Интенсивность сигнала ФЛ нормирована на интенсивность ли- 


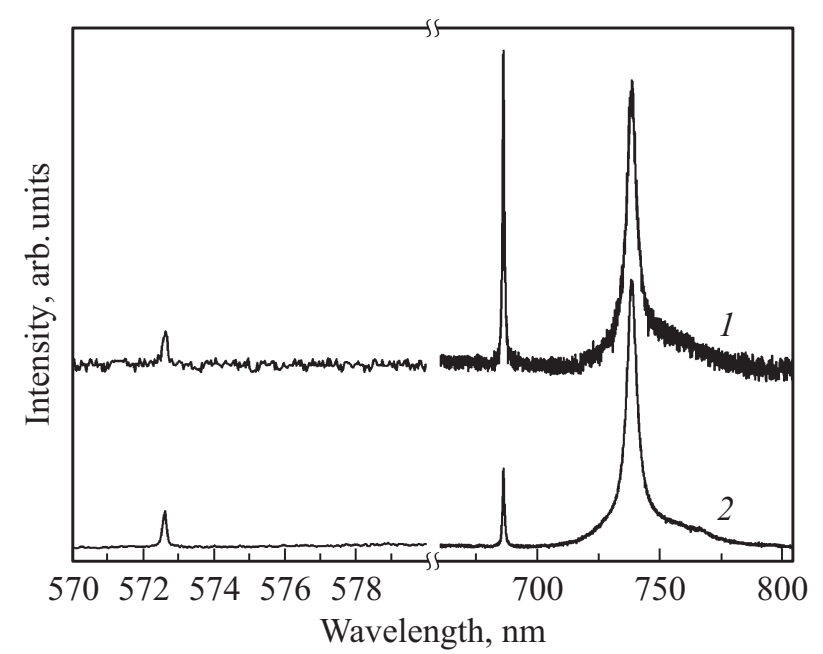

Рис. 2. Спектры фотолюминесценции ансамбля наноалмазов NDE1 при мощности возбуждающего лазера $1-0.04 \mathrm{~mW}$ и $2-1 \mathrm{~mW}$. Спектры измерены при $T=300 \mathrm{~K}$ и нормированы на рамановскую линию алмаза $(572.5 \mathrm{~nm})$.

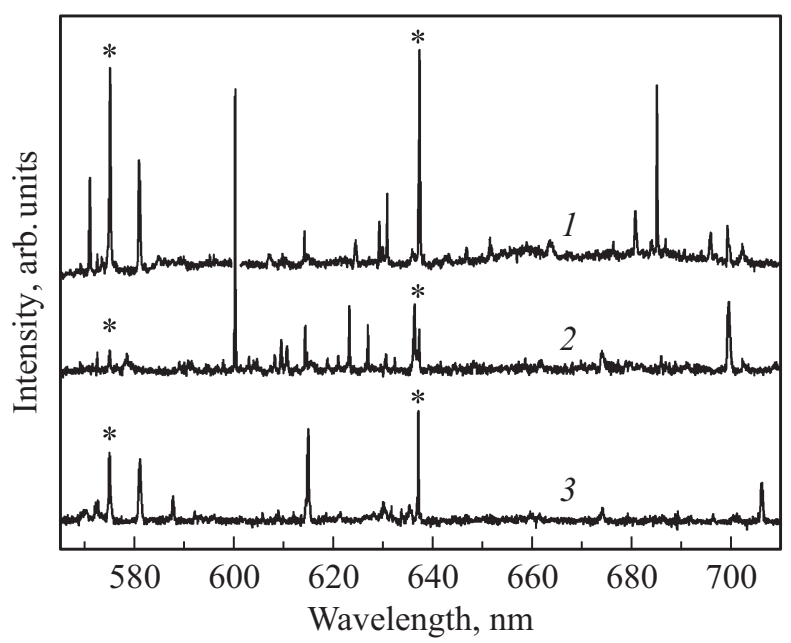

Рис. 3. Спектры ФЛ трех различных ансамблей наноалмазов $(1-\mathrm{NDE} 4,2-\mathrm{NDE} 5,3-\mathrm{NDE6})$ при $T=77 \mathrm{~K}$. Линии, соответствующие оптическим центрам окраски $N V^{0}$ и $N V^{-}$ обозначены звездочками.

нии $1332.5 \mathrm{~cm}^{-1}(572.5 \mathrm{~nm})$, которая является линией рамановского рассеяния, соответствующего трехкратно вырожденному ТО фонону в алмазе [40]. FWHM этой линии, измеренная с использованием спектрометра с тройным монохроматором, обеспечивающего спектральное разрешение $0.35 \mathrm{~cm}^{-1}$, составляет $\sim 2.5 \mathrm{~cm}^{-1}$. Соотношение интенсивностей линий $686 \mathrm{~nm}$ и $738 \mathrm{~nm}$ зависит от мощности накачки. При мощности лазера $0.04 \mathrm{MBT}$ интенсивность линии $686 \mathrm{~nm}$ больше интенсивности линии $738 \mathrm{~nm}$ центра SiV. При увеличении мощности лазера до $1 \mathrm{~mW}$ сдвиг спектрального положения и уширение БФЛ центра $\mathrm{SiV}$ не наблюдается. Линия $686 \mathrm{~nm}$ практически не изменяет спектрального положения своего максимума, а ее FWHM увеличивается с $0.89 \mathrm{~nm}$ до $1 \mathrm{~nm}$.
На рис. 3 показаны спектры ФЛ трех различных ансамблей наноалмазов (NDE4-NDE6) при $T=77 \mathrm{~K}$. Наиболее интенсивные узкие линии на рис. 3 (FWHM указана в скобках) соответственно $571.04(0.25) \mathrm{nm}$, $575.13(0.29) \mathrm{nm}, 581(0.47) \mathrm{nm}, 630.82(0.2) \mathrm{nm}, 637.35$ $(0.3) \mathrm{nm}, 680.67(0.5) \mathrm{nm}$ и $685(0.2) \mathrm{nm}$ для NDA4; 600.3 $(0.15) \mathrm{nm}, 623.27 \quad(0.17) \mathrm{nm}, 626.95(0.21) \mathrm{nm}, 636.27$ $(0.5) \mathrm{nm}, 637.3(0.25) \mathrm{nm}, 699.38(0.65) \mathrm{nm}$ для NDA5; $575.05(0.45) \mathrm{nm}, 614.9(0.38) \mathrm{nm}, 637.06(0.19) \mathrm{nm}$, $706.06(0.54) \mathrm{nm}$ для NDA6. По сравнению с комнатной температурой в спектрах ФЛ при азотной температуре наблюдается больше узких линий в исследованном спектральном диапазоне и уменьшение их ширин. Во всех трех спектрах наблюдаются узкие линии вблизи 575 и $637 \mathrm{~nm}$, соответствующие оптическим центрам окраски соответственно $N V^{0}$ и $N V^{-}$(обозначены звездочками на рис. 3). Из рис. 1 и 3 видно, что с понижением температуры до $T=79 \mathrm{~K}$ увеличилось количество регистрируемых узких линий в спектрах. Можно предположить, что новые наблюдаемые линии связаны с эмиттерами, имеющими различные вероятности излучательных переходов и активационные энергии безызлучательных переходов. ФЛ эмиттеров с малыми вероятностями излучательных переходов и малыми активационными энергиями безызлучательных переходов детектируются только при низкой температуре.

Температурная зависимость сигнала ФЛ ансамбля наноалмазов NDE4 в спектральном интервале $570-590 \mathrm{~nm}$ и в диапазоне температур 79-300 K представлена на рис. 4. При росте температуры от $79 \mathrm{~K}$ до $300 \mathrm{~K}$ для всех линий ФЛ наблюдается длинноволновый сдвиг, увеличение ширины, а также имеет место температурное гашение их интенсивности.

На рис. 5 представлены спектры ФЛ ансамбля наноалмазов NDE5 при $T=79,140$ и $220 \mathrm{~K}$ в ближней инфракрасной области спектра. Узкая линия $699.4 \mathrm{~nm}$ с ростом

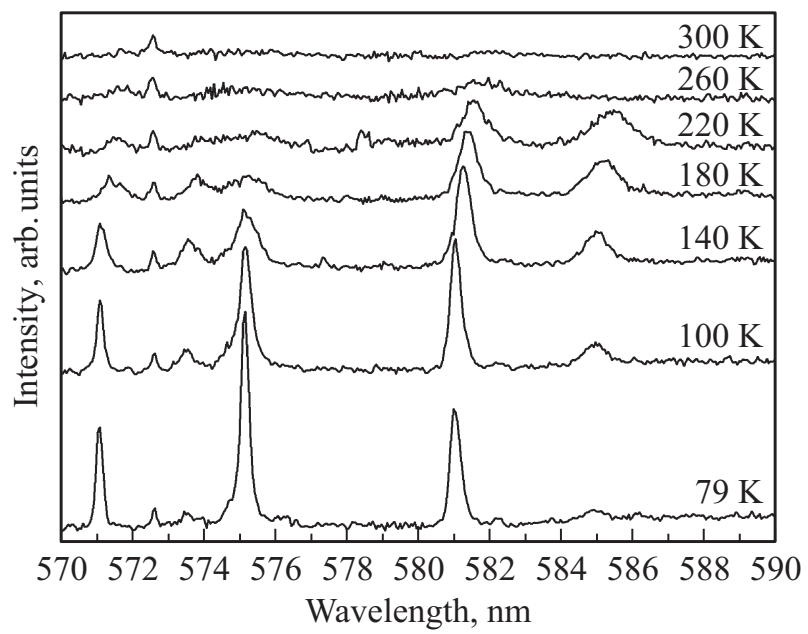

Рис. 4. Спектры фотолюминесценции ансамбля наноалмазов NDE4, измеренные в диапазоне температур $T=79-300 \mathrm{~K}$. Спектры нормированы на интенсивность рамановской линии алмаза $572.5 \mathrm{~nm}$. 


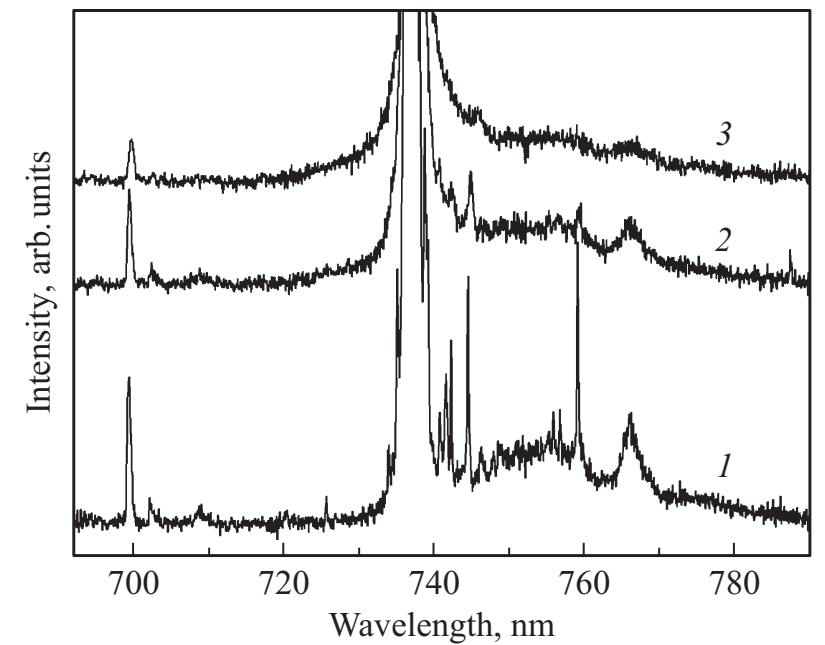

Рис. 5. Спектры ФЛ ансамбля наноалмазов NDE5 при температуре: 1 - $79 \mathrm{~K}, 2-140 \mathrm{~K}, 3-220 \mathrm{~K}$. Спектры нормированы на интенсивность рамановской линии алмаза.

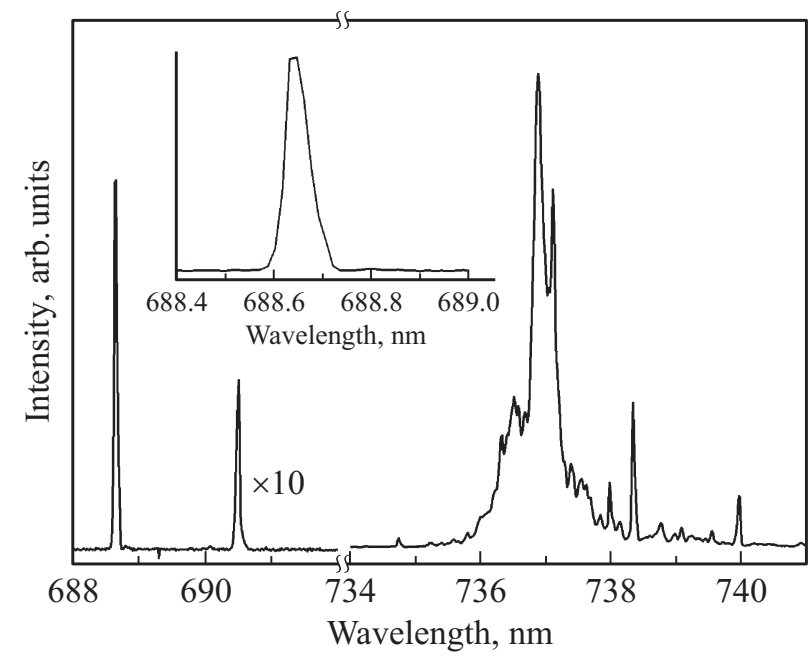

Рис. 6. Спектры ФЛ ансамбля наноалмазов при $T=10 \mathrm{~K}$. На вставке показан спектр ФЛ линии $688.64 \mathrm{~nm}$ в увеличенном масштабе.

температуры сдвигается в длинноволновую область и уширяется, ее интенсивность падает относительно интенсивности БФЛ центров $\mathrm{SiV}$. В спектральной области БФЛ центров $\mathrm{SiV}$ при $T=79 \mathrm{~K}$ наблюдаются узкие линии (FWHM линий указана в скобках) $738.8(0.25) \mathrm{nm}$, $742.2(0.2) \mathrm{nm}, 744.52(0.3) \mathrm{nm}$ и $759.1(0.2) \mathrm{nm}$. Эти линии могут быть обусловлены одиночными центрами $\mathrm{SiV}$ в областях с различными внутренними напряжениями в наноалмазах $[11,13]$. При $T=79 \mathrm{~K}$ интенсивности узких линий в области фононного крыла БФЛ центра $\mathrm{SiV}$ сравнимы с интенсивностью узкой линии $699.4 \mathrm{~nm}$, но с ростом температуры они затухают сильнее. В спектрах ФЛ NDE5 при $T=79 \mathrm{~K}$ регистрируются полосы $756 \mathrm{~nm}$ и $766 \mathrm{~nm}$, обусловленные локальными колебательными модами [41].
Фрагменты спектра ФЛ наноалмазов, измеренные при $T=10 \mathrm{~K}$, приведены на рис. 6 . В спектрах наблюдается БФЛ ансамбля центров $\mathrm{SiV}$ с максимумом на длине волны $\sim 736.8 \mathrm{~nm}$. В соответствие с четырехуровневой электронной структурой центра $\mathrm{SiV}$ его БФЛ при температуре жидкого гелия имеет тонкую структуру и состоит из четырех линий, образовавшихся из-за расщепления основного и возбужденного состояний центра $\mathrm{SiV}[11,13,16-18,41]$. Неоднородное уширение изза присутствия остаточных напряжений в наноалмазе приводит к замыванию линий тонкой структуры $[11,13]$. БФЛ ансамбля центров $\mathrm{SiV}$ является суперпозицией БФЛ одиночных центров $\mathrm{SiV}$, находящихся в областях наноалмаза с различными внутренними напряжениями. Следствием распределения напряжений в наноалмазе являются различные спектральные положения БФЛ центров $\mathrm{SiV}$ [16]. Присутствие одиночных узких линий в области длин волн больше $738 \mathrm{~nm}$ связано центрами $\mathrm{SiV}$ в областях наноалмаза с различными напряжениями [34]. В спектре на рис. 6 наблюдаются две такие линии на длинах волн $738.33 \mathrm{~nm}$ и $739.96 \mathrm{~nm}$ с FWHМ соответственно $0.045 \mathrm{~nm}$ и $0.055 \mathrm{~nm}$.

На рис. 6 приведены также две узкие линии $688.64(0.055) \mathrm{nm}$ и $690.5(0.061) \mathrm{nm}$ (в скобках указана их FWHM) при $T=10 \mathrm{~K}$. Наблюдаемое уменьшение ширин линий при понижении температуры (с 1-2 nm при комнатной температуре до $0.055 \mathrm{~nm}$ при $T=10 \mathrm{~K}$ ) связано с уменьшением влияния однородного уширения [13]. Можно предположить, что при $T=10 \mathrm{~K}$ основной причиной уширения линий является неоднородное уширение.

В спектрах ФЛ центров окраски в алмазе электронфононное взаимодействие приводит к появлению длинноволнового крыла в спектре БФЛ (см. линию $\mathrm{SiV}$ на рис. 5). В спектрах обнаруженных узких линий ФЛ фононное крыло не наблюдается, что указывает на слабое электрон-фононное взаимодействие. Спектральные положения наблюдаемых новых линий не позволяют приписать их какому-либо известному в алмазе центру окраски [42]. Мы не располагаем надежными основаниями, чтобы сделать вывод о том, какие примесные атомы входят в состав точечных дефектов и какова структура дефектов, которые ответственны за обнаруженные линии ФЛ. Возможно, эти точечные дефекты имеют различные структурные конфигурации и сильно чувствительны к локальным напряжениям.

\section{4. Выводы}

В работе исследованы спектральные характеристики обнаруженных узких линий ФЛ ансамблей наноалмазов, полученных методом реактивного ионного травления в кислородной плазме алмазных частиц, синтезированных методом химического газофазного осаждения на кремниевой подложке. Исследованные наноалмазы характеризуются узкой рамановской линией алмаза $1332.5 \mathrm{~cm}^{-1}$, ши- 
рина которой при комнатной температуре не превышает $2.5 \mathrm{~cm}^{-1}$. Новые узкие линии наблюдались в спектрах ФЛ наноалмазов при практически полном отсутствии фонового сигнала в виде широкой бесструктурной полосы, характерной для гетероэпитаксиальных алмазов газофазного синтеза. Отсутствие у узких линий фононного крыла свидетельствует о слабом электрон-фононном взаимодействии. С ростом температуры обнаруженные линии ФЛ испытывают сдвиг в длинноволновую область, сопровождающийся увеличением их ширины и температурным гашением. FWHM линий увеличивается с $0.2-0.6 \mathrm{~nm}$ при $T=77 \mathrm{~K}$ до $1-2 \mathrm{~nm}$ при $T=300 \mathrm{~K}$. При температуре $T=10 \mathrm{~K}$ минимальная обнаруженная ширина линий составила $0.055 \mathrm{~nm}$.

\section{Список литературы}

[1] J. Orwa, A. Greentree, I. Aharonovich, A. Alves, J. Van Donkelaar, A. Stacey, S. Prawer. J. Lumin. 130, 1646 (2010).

[2] S. Pezzagna, D. Rogalla, D. Wildanger, J. Meijer, A. Zaitsev. New J. Phys. 13, 035024 (2011).

[3] I. Aharonovich, A.D. Greentree, S. Prawer. Nature Photon. 5, 397 (2011).

[4] K. Beha, H. Fedder, M. Wolfer, M.C. Becker, P. Siyushev, M. Jamali, A. Batalov, C. Hinz, J. Hees, L. Kirste, H. Obloh, E. Gheeraert, B. Naydenov, I. Jakobi, F. Dolde, S. Pezzagna, D. Twittchen, M. Markham, D. Dregely, H. Giessen, J. Meijer, F. Jelezko, C.E. Nebel, R. Bratschitsch, A. Leitenstorfer, J. Wrachtrup. Beilstein J. Nanotechnol. 3, 895 (2012).

[5] M.W. Doherty, N.B. Manson, P. Delaney, F. Jelezko, J. Wrachtrup, L.C. Hollenberg. Phys. Rep. 528, 1 (2013).

[6] I. Aharonovich, E. Neu. Adv. Opt. Mater. 2, 911 (2014).

[7] A. Nagl, S.R. Hemelaar, R. Schirhagl. An. Bioanal. Chem. 407, 7521 (2015).

[8] A. Neves, M.H. Nazaré. Properties, growth and applications of diamond. IET, London (2001). $427 \mathrm{p}$.

[9] I.I. Vlasov, A.S. Barnard, V.G. Ralchenko, O.I. Lebedev, M.V. Kanzyuba, A.V. Saveliev, V.I. Konov, E. Goovaerts. Adv. Mater. 21, 808 (2009).

[10] A. Basov, M. Rähn, M. Pärs, I. Vlasov, I. Sildos, A. Bolshakov, V. Golubev, V. Ralchenko. Phys. Status Solidi A. 206, 2009 (2009).

[11] E. Neu, C. Arend, E. Gross, F. Guldner, C. Hepp, D. Steinmetz, E. Zscherpel, S. Ghodbane, H. Sternschulte, D. Steinmüller-Nethl, Y. Liang, A. Krueger, C. Becher. Appl. Phys. Lett. 98, 243107 (2011).

[12] S.A. Catledge, S. Singh. Adv. Sci. Lett. 4, 512 (2011).

[13] E. Neu, C. Hepp, M. Hauschild, S. Gsell, M. Fischer, H. Sternschulte, D. Steinmüller-Nethl, M. Schreck, C. Becher. New J. Phys. 15, 043005 (2013).

[14] B. Pingault, J.N. Becker, C.H. Schulte, C. Arend, C. Hepp, T. Godde, A.I. Tartakovskii, M. Markham, C. Becher, M. Atatüre. Phys. Rev. Lett. 113, 263601 (2014).

[15] С.А. Грудинкин, Н.А. Феоктистов, К.В. Богданов, М.А. Баранов, А.В. Баранов, А.В. Федоров, В.Г. Голубев. ФТП 48, 283 (2014).

[16] C. Arend, J.N. Becker, H. Sternschulte, D. Steinmüller-Nethl, C. Becher. Phys. Rev. B. 94, 045203 (2016).

[17] K. Li, Y. Zhou, A. Rasmita, I. Aharonovich, W. Gao. Phys. Rev. Appl. 6, 024010 (2016).
[18] J.L. Zhang, H. Ishiwata, T.M. Babinec, M. Radulaski, K. Müller, K.G. Lagoudakis, C. Dory, J. Dahl, R. Edgington, V. Soulière, G. Ferro, A.A. Fokin, P.R. Schreiner, Z.-X. Shen, N.A. Melosh, J. Vučković. Nano Lett. 16, 212 (2015).

[19] L. Himics, S. Tóth, M. Veres, M. Koós. Opt. Quant. Electron. 48, 394 (2016).

[20] C. Arend, P. Appel, J.N. Becker, M. Schmidt, M. Fischer, S. Gsell, M. Schreck, C. Becher, P. Maletinsky, E. Neu. Appl. Phys. Lett. 108, 063111 (2016).

[21] J. Rabeau, Y. Chin, S. Prawer, F. Jelezko, T. Gaebel, J. Wrachtrup. Appl. Phys. Lett. 86, 131926 (2005).

[22] E. Wu, J. Rabeau, G. Roger, F. Treussart, H. Zeng, P. Grangier, S. Prawer, J.-F. Roch. New J. Phys. 9, 434 (2007).

[23] M. Wolfer, A. Kriele, O. Williams, H. Obloh, C.-C. Leancu, C. Nebel. Phys. Status Solidi A. 206, 2012 (2009).

[24] S. Castelletto, A. Edmonds, T. Gaebel, J. Rabeau. IEEE J. Sel. Top. Quant. Electron. 18, 1792 (2012).

[25] S. Tóth, L. Himics, M. Koós. J. Lumin. 176, 367 (2016).

[26] I. Aharonovich, S. Castelletto, D.A. Simpson, A. Stacey, J. McCallum, A.D. Greentree, S. Prawer. Nano Lett. 9, 3191 (2009).

[27] I. Aharonovich, S. Castelletto, D. Simpson, A. Greentree, S. Prawer. Phys. Rev. A. 81, 043813 (2010).

[28] S. Castelletto, A. Boretti. Opt. Lett. 36, 4224 (2011).

[29] A. Stacey, D.A. Simpson, T.J. Karle, B.C. Gibson, V.M. Acosta, Z. Huang, K.M.C. Fu, C. Santori, R.G. Beausoleil, L.P. McGuinness, K. Ganesan, S. Tomljenovic-Hanic, A.D. Greentree, S. Prawer. Adv. Mater. 24, 3333 (2012).

[30] S.A. Momenzadeh, F.F. de Oliveira, P. Neumann, D.B. Rao, A. Denisenko, M. Amjadi, Z. Chu, S. Yang, N.B. Manson, M.W. Doherty, J. Wrachtrup. Phys. Rev. Appl. 6, 024026 (2016).

[31] R.G. Sandstrom, O. Shimoni, A.A. Martin, I. Aharonovich. Appl. Phys. Lett. 105, 181104 (2014).

[32] D.G. Monticone, P. Traina, E. Moreva, J. Forneris, P. Olivero, I. Degiovanni, F. Taccetti, L. Giuntini, G. Brida, G. Amato, M. Genovese. New J. Phys. 16, 053005 (2014).

[33] K. Bray, R. Sandstrom, C. Elbadawi, M. Fischer, M. Schreck, O. Shimoni, C. Lobo, M. Toth, I. Aharonovich. ACS Appl. Mater. Interfaces. 8, 7590 (2016).

[34] S.A. Grudinkin, N.A. Feoktistov, M.A. Baranov, A.N. Smirnov, V.Y. Davydov, V.G. Golubev. Nanotechnol. 27, 395606 (2016).

[35] Н.А. Феоктистов, В.И. Сахаров, И.Т. Серенков, В.А. Толмачев, И.В. Коркин, А.Е. Алексенский, А.Я. Вуль, В.Г. Голубев. ЖТФ 81, 132 (2011).

[36] A.E. Aleksenskiy, E.D. Eydelman, A.Y. Vul. Nanosci. Nanotechnol. Lett. 3, 68 (2011).

[37] S.A. Grudinkin, N.A. Feoktistov, A.V. Medvedev, K.V. Bogdanov, A.V. Baranov, A.Y. Vul, V.G. Golubev. J. Phys. D. 45, 062001 (2012).

[38] V. Sedov, A. Khomich, V. Ralchenko, A. Martyanov, S. Savin, O. Poklonskaya, N. Trofimov. J. Coat. Sci. Technol. 2, 38 (2015).

[39] L. Bergman, M. McClure, J. Glass, R. Nemanich. J. Appl. Phys. 76, 3020 (1994).

[40] S. Prawer, R.J. Nemanich. Philosophical Transactions of the Royal Society of London A: Mathematical, Phys. Eng. Sci. 362, 2537 (2004).

[41] H. Sternschulte, K. Thonke, R. Sauer, P. Münzinger, P. Michler. Phys. Rev. B. 50, 14554 (1994).

[42] A.M. Zaitsev. Optical properties of diamond: a data handbook, Springer Science \& Business Media, Berlin (2013). 502 p. 